\title{
RELACIÓN ENTRE ENFERMEDAD PERIODONTAL Y DIABETES MELLITUS TIPO II. REVISIÓN DE LA LITERATURA
}

\section{RELATIONSHIP BETWEEN PERIODONTAL DISEASE AND DIABETES. LITERATURE REVIEW}

\author{
Cruz Alexander ${ }^{1 a}$, Carrera Alex ${ }^{1 a}$, Rivera Gabriela ${ }^{1 a}$, Núñez Alejandra ${ }^{2 c}$, Paulina Mantilla ${ }^{1 a}$, Armas Ana del Carmen ${ }^{1 b c}$
}

\section{RESUMEN}

La diabetes es una de las patologías más frecuentes con repercusiones a nivel sistémico, irreversibles y con alta prevalencia en el mundo. En la actualidad en el Ecuador, existe un promedio de 800.000 diabéticos, de los cuales 100.000 se encuentran en tratamiento, según las autoridades de salud y la Fundación Diabetes Juvenil del Ecuador. Así la presente revisión busca actualizar los conocimientos, en el área de la salud, tanto en medicina como en odontología, obligándonos a contar con una revisión de datos actuales de la relación entre la enfermedad periodontal y la diabetes mellitus tipo II , y cómo estas dos patologías, pueden ir de la mano y afectar el medio bucal y sistémico, relacionándolos con los cambios histológicos y mediadores químicos en el medio bucal que se ven acentuados por la presencia de enfermedad periodontal y diabetes, ya sea esta, controlada o no. Fueron revisadas bases de datos en fuentes bibliográficas de Pubmed y SclELO, colocando como límites de tiempo del 2016 hasta el 2017, empleando como palabras clave "cavidad bucal", "diabetes", "enfermedad periodontal" y sus similares en inglés, se obtuvieron 74 artículos, de los mismos se seleccionaron 12 que fueron analizados y sirvieron de base para la ejecución de esta revisión. Después de la revisión exhaustiva de los artículos, la mayoría de los estudios revisados (7 artículos), reportaron una relación entre las dos patologías, las cuales van de la mano, acentuándose en personas mayores. Se concluye que entre la enfermedad periodontal y la diabetes tipo II existe una relación estrecha, obteniendo mayor prevalencia de enfermedad periodontal, células inflamatorias en presencia de diabetes tipo II con un mal control glicémico.

Palabras Clave: Alteraciones; Cavidad bucal; Diabetes; enfermedad periodontal. (Fuente: DeCS BIREME)

\section{ABSTRACT}

Diabetes is one of the most frequent pathologies with repercussions at the systemic level, irreversible and with high prevalence in the world. Currently in Ecuador, there is an average of 800,000 diabetics, of whom 100,000 are in treatment, according to health authorities and the Juvenile Diabetes Foundation of Ecuador. This review seeks to update knowledge in the area of health, both in medicine and in dentistry, forcing us to have a review of current data on the relationship between periodontal disease and type II diabetes mellitus, and how These two pathologies can go hand in hand and affect the oral and systemic environment, relating them to the histological changes and chemical mediators in the oral environment that are accentuated by the presence of periodontal disease and diabetes, whether controlled or not. Databases were revised in Pubmed and SclELO bibliographic sources, placing as time limits from 2016 to 2017, using as key words "oral cavity", "diabetes", "periodontal disease" and its similar in English, 74 articles were obtained, of which 12 were selected that were analyzed and served as the basis for the execution of this review. After the exhaustive review of the articles, most of the studies reviewed ( 7 articles), reported a relationship between the two pathologies, which go hand in hand, accentuating in older people. It is concluded that between periodontal disease and type II diabetes there is a close relationship, obtaining a higher prevalence of periodontal disease, inflammatory cells in the presence of type II diabetes with poor glycemic control.

Keywords: Disorders; oral cavity; Diabetes; periodontal disease. (Source: MeSH NLM)

Recibido: 12 de diciembre de 2017

${ }^{1}$ Universidad Tecnológica Equinoccial. Ecuador

${ }^{2}$ Universidad Central del Ecuador. Ecuador

${ }^{a}$ Odontólogo. ${ }^{b}$ Profesora de la Carrera de Odontología. ${ }^{c} \mathrm{PhD}$ en Operatoria Dental Correspondencia:

Alex Cruz

Correo electrónico: alexandercruz1993@hotmail.com
Este es un artículo Open Access distribuido bajo la licencia Creative Commons Atribución-NoComercial- Compartir Igual 4.0

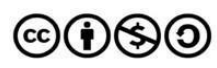

Citar como: Cruz A, Carrera A, Rivera G, Núñez A, Armas A. Relación entre enfermedad periodontal y diabetes mellitus tipo ii. Revisión de la literatura. kiru. 2018; 15(3): 154 - 158. https://doi.org/10.24265/kiru.2018.v15n3.08 


\section{INTRODUCCIÓN}

La diabetes es una enfermedad crónica que se desencadena cuando el páncreas no produce suficiente insulina, una hormona que regula el nivel de azúcar, o glucosa, en la sangre, e incluso cuando el organismo no puede utilizar con eficacia la insulina que produce ${ }^{(1)}$. En los últimos 20 años, el rápido crecimiento de los índices de obesidad ha generado un importante aumento de la prevalencia de diabetes y síndrome metabólico (1). Observándose incremento notable de ambas enfermedades, es posible que la muerte y la discapacidad por este tipo de enfermedades empiecen a aumentar de nuevo en países en vías de desarrollo ${ }^{(2)}$. La diabetes constituye una patológica difícil de detectar en periodos iniciales, en países como Estados Unidos se estima que cerca de un cuarto de los pacientes diabéticos no han sido diagnosticados ${ }^{(3)}$. Evidencias muestran que el diagnóstico temprano de la diabetes puede mitigar sus complicaciones y mejorar el estado de salud de los pacientes (4). Por otro lado, la pre existencia de periodontitis puede provocar una peor evolución de enfermedades renales y cardiovasculares ${ }^{(5-6)}$.

En el proceso de la enfermedad periodontal, los tejidos blandos y estructuras de soporte del diente, se ven severamente afectadas, por naturaleza inflamatoria e infecciosa ${ }^{(7)}$, que desencadena inflamación gingival conocida como gingivitis, que es considerada una etapa inicial de la enfermedad periodontal, y que puede llegar hasta un proceso inflamatorio que afecta a los tejidos periodontales profundos donde se produce destrucción del hueso y ligamento que soportan y sujetan los dientes, denominado periodontitis, ya sea esta aguda o

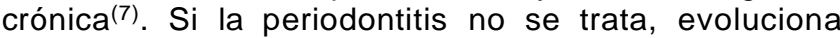
destruyendo todo el soporte del diente y con ello la pérdida del mismo ${ }^{(8)}$.

Debemos señalar que, aunque la presencia de ciertas bacterias específicas es necesaria para que ocurra la periodontitis, no es suficiente, porque la respuesta del huésped a los patógenos periodontales es la responsable de la destrucción de los tejidos ${ }^{(9)}$. Esto explica por qué muchos individuos pueden albergar los microorganismos sin desarrollar la enfermedad ${ }^{(9)}$.

Una de las primeras manifestaciones clínicas de la enfermedad periodontal es la aparición de bolsas periodontales, las cuales condicionan un lugar favorable para la colonización bacteriana (10). Para el diagnostico se realiza un examen clínico con ayuda de una sonda periodontal, la cual sirve para determinar la profundidad de las bolsas. Esto puede complementarse con un examen radiológico, para observar la pérdida ósea y un examen microbiológico para analizar el tipo de agentes infecciosos que se encuentran en la zona ${ }^{(10)}$.

Existen factores de riesgo para las periodontitis que pueden ser modificables, intervenidos o controlados para reducir el riesgo de iniciación o progresión de la enfermedad periodontal, como los factores de comportamiento o estilo de vida por ejemplo el tabaquismo, los niveles de bacterias patógenas específicas y la diabetes mellitus (11). Se ha demostrado científicamente que la diabetes mal controlada del tipo 1 y tipo 2, es el factor de riesgo con mayor influencia en la progresión y desarrollo de la periodontitis ${ }^{(12)}$, puesto que en la diabetes se promueve la alteración de la función de neutrófilos y la deposición en los tejidos periodontales de los productos finales derivados de la glicolización avanzada (13-14).

Los profesionales de la salud bucal tienen una gran oportunidad de identificar diabetes o prediabetes no diagnosticada, en un paciente que acude a la consulta odontológica, el cual sin ningún problema puede referir a un especialista o médico, de ahí la importancia de la relación Médico-Odontólogo (15). Así la presente revisión de la literatura busca actualizar los conocimientos sobre la relación entre diabetes y enfermedad periodontal, teniendo como recurso a la información actualizada sobre dicha relación.

\section{MATERIALES Y MÉTODOS}

Se planteó una búsqueda bibliográfica en las bases de datos PudMed y Scielo, usando como palabras clave: "cavidad Oral", "Diabetes", "enfermedad periodontal" y sus homólogos en ingles de los últimos 2 años (Período comprendido entre 2016 y 2017), en las cadenas de búsqueda empleadas combinamos las palabras claves con los conectores booleanos AND Y OR. Empleando también literatura impresa considerada obligatoria de ser citada.

Los criterios de inclusión para el levantamiento de artículos fueron estudios de casos, ensayos clínicos, metanálisis, revisiones de literatura y estudios in vitro que presenten una evaluación de la relación entre diabetes tipo 2 y periodontitis, incluyendo datos estadísticos de dicha comparación.

Los criterios de exclusión fueron aquellos artículos en los cuales luego de la lectura pertinente realizada por los autores de la presente investigación, se verificó que no contienen la información requerida. Se obtuvieron, 74 artículos, después de la lectura y exclusión de 62 artículos, la búsqueda arrojó 12 artículos 
considerándolos que proporcionaban datos de mayor relevancia para la revisión planteada. La cual se realizó en octubre del 2017.

\section{RESULTADOS}

La relación entre la diabetes tipo II y la enfermedad periodontal abarca una serie de datos experimentales que demuestran que la diabetes es un factor de riesgo de gingivitis y periodontitis. La diabetes mellitus fomenta al incremento de la prevalencia, severidad y progresión de la periodontitis ${ }^{(13)}$.

Se ha realizado un cuadro comparativo que indica el resultado de cada autor en los artículos revisados (ver tabla1).

Tabla 1. Cuadro de resumen de estudios realizados para evaluar relación entre enfermedad periodontal y diabetes tipo II

Autor

Objetivo

Ramos et al., (2) Ensayo clínico (2017)

Ziukaite et al.,(16) Ensayo Clinico (2017)
Identificar la presencia de bacilos negro pigmentantés (BNP) en bolsas periodontales de pacientes diabéticos tipo II y determinar una relación con su control glicémico.

Evaluar la prevalencia de diabetes entre los pacientes con periodontitis y evaluar si la diabetes está relacionada con el grado y la gravedad de la periodontitis.

Revisar la efectividad de la terapia periodontal o no, tiene una relación con la Yitayeh (17) Revisión sistemática meta-analisis (2017)

Li y Li (18). Estudio in vitro (2016)

\section{disminución de HbA1c y} nivel glicémico en diabetes tipo II

Evaluar el efecto de valores altos de glucosa en la proliferación de células del

Materiales y métodos

Determinar las condiciones periodontales en pacientes con diagnóstico de diabetes mellitus tipo II.
Descriptivo, transversal, muestra de 202 individuos con diabetes mellitus II, se realizó control sobre el uso de insulina externa $u$ otros medicamentos, se pidió la última glicemia y HbA1c mediante análisis de laboratorio, se realizó una evaluación periodontal (profundidad de sondaje, movilidad, nivel de inserción, características de la encía) de todos los dientes presentes en boca.

Descriptivo, longitudinal, correlacional, 20 pacientes con diabetes mellitus II, se realizó una historia clínica periodontal y se envió muestras de sangre (se realizó HbA1c) y placa dental (cultivo)

Estudio retrospectivo correlacional, Evaluaron la información de 5375 pacientes con enfermedad periodontal, con y sin diabetes ligamentos periodontal in vitro.
Estudios entre 2005 y 2015 en 4 bases de datos y búsqueda manual. periodontal por $1,4,7,10,14$ y 17 en concentraciones normal $y$ alto de glucosa y MMT.
Se cultivó células de ligamento

Conclusión/Hallazgos

Se encontró alta prevalencia de enfermedad periodontal en pacientes con diabetes tipo II, sin diferencia significativa entre pacientes

controlados y no controlados

0.970

No existió una relación estadísticamente significativa entre enfermedad periodontal, periodontopatógenos y diabetes tipo II, aunque los pacientes tienen una alta prevalencia de periodontitis

No se pudo establecer una relación entre diabetes y enfermedad periodontal. Obteniendo solo el $3.7 \%$ de prevalencia de diabetes en pacientes con enfermedad periodontal.

7 estudios controlados randomizados envolviendo 940 participantes. Existió reducción significativa de la $\mathrm{HbA} 1 \mathrm{c}$ y el nivel de glucosa plasmático en ayunas en pacientes con diabetes tipo II y enfermedad periodontal con tratamiento periodontal no quirúrgico. Existió una inhibición de la proliferación de las células del ligamento periodontal. 
Kaur et al., (19) Ensayo clínico (2016)

Kapellas et al.,(20) Ensayo clínico (2016)

Eke et al.,(21) Ensayo clínico (2016)

Khanuja et al., (22) Ensayo clínico (2016)

al., (23)
(2017)

Ensayo clínico

Miranda et al., (24) (2016)

Ensayo Clínico

Bhavsar et al., (24) (2016)

Ensayo clínico
Investigar la relación entre hemoglobina glicosilada $y$ complicaciones diabéticas con periodontitis crónica.

Reportar el efecto de la terapia periodontal sobre el control glicémico entre personas con obesidad.

Determinar los perfiles de riesgo de la población promedio para periodontitis severa y no severa en adultos estadounidenses usando medidas de vigilancia óptimas y definiciones de caso estándar.

Investigar la relación entre hemoglobina glicosilada y complicaciones de la diabetes con periodontitis crónica.

Comparar los parámetros de inflamación periodontal y peri-implantar con grupo de prediabetes, diabetes tipo II y pacientes sanos.

Evaluar la influencia del control glicémico en los niveles y frecuencia de los periodontopatógenos en pacientes con diabetes tipo II.

Evaluación de los niveles de glucosa séricos en el líquido crevicular de pacientes con o sin diabetes del examen periodontal y sangre venosa.
Estudio transversal. Pacientes con diabetes mellitus tipo II controlados, con diagnóstico periodontal, examen de HcA1b y glucosa plasmática en ayunas. Y se comparó con un grupo de 67 personas con salud periodontal.

62 participantes con diabetes recibieron un tratamiento periodontal en una cita y se realizó examen HbA1c, CRP y estado periodontal después de 3 meses de intervención.

Se utilizó los datos entre 2009-2012 de la encuesta de examinación nacional de salud y nutrición, con los adultos mayores de 30 años y se identificó la enfermedad periodontal y la prevalencia de algunas enfermedades y factores de riesgo.

207 pacientes con diabetes y enfermedad periodontal se les examinó periodontalmente toda la boca, un grupo control de 67 personas periodontalmente sanos se realizó HbAic, FPG y PPG en ambos grupos.

45 pacientes con prediabetes, 43 pacientes con diabetes tipo II y 42 del grupo control, se realizó un examen periodontal completo, y evaluación de HbAc1 y se comparó resultados.

56 pacientes con periodontitis crónica y diabetes tipo II se les asignó en dos grupos según su HbA1c: uno menor de $<8 \%$ y otro igual o $>$ de $8 \%$. Se comparó los niveles de bacterias mediante un cultivo.

70 pacientes, 35 con diabetes y 35 sin diabetes se les evaluó sangre de la encía más afectada por la enfermedad periodontal, y se evaluó los niveles séricos al igual que en la sangre venosa.
Se encontró asociación entre el control glicémico y las complicaciones diabéticas con periodontitis. En todos los aspectos revisados.

El tratamiento periodontal no quirúrgico no dio una relación significativa en la reducción de HbA1c.

$46 \%$ de la población tuvo periodontitis, la mayor probabilidad de periodontitis en adultos mayores y en hombres con diabetes no controlada.

Existe una asociación entre el control glicémico y las complicaciones diabéticas con las periodontitis.

Los parámetros inflamatorios de los pacientes con pre diabetes y con diabetes tipo II fueron mayores en comparación con los de controles.

Se concluyó que un control glicémico malo, influye en los niveles y frecuencia de los periodontopatógenos en el biofilm subgingival en pacientes con diabetes tipo II y periodontitis crónica.

Los niveles séricos de glucosa medidos en la examinación periodontal pueden ser un excelente recurso para la estimación de glucosa en la sangre.
Las diferentes implicaciones existentes a nivel bucal en pacientes afectados por Diabetes tipo II sumado al elevado porcentaje de individuos de diferente edad y condición social afectados con esta enfermedad, obligan tanto a la academia como a los diferentes grupos de investigación a realizar estudios frecuentes y revisiones de lo que en la literatura se reporta sobre el tema, con el propósito de mantener informado al profesional odontólogo sobre este tema buscando proporcionar un tratamiento integral al paciente afectado por esta dolencia.
Es de vital importancia que el especialista y médico general continuamente actualicen sus conocimientos para manejar de una manera integral a sus pacientes, por este motivo se realizó esta revisión consultando las mejores bases de datos e información más actualizada. Después del análisis correspondiente, en los últimos 2 años, se llegó a la conclusión que existe un aumento de la severidad de la enfermedad periodontal en presencia de diabetes tipo II, teniendo una relación entre las dos, aunque esto debería ser aclarado con estudios clínicos aleatorizados verificando los resultados. 


\section{CONCLUSIÓN}

Entre la enfermedad periodontal y la diabetes tipo II existe una relación estrecha, obteniendo mayor prevalencia de periodontitis, células inflamatorias en presencia de diabetes tipo II con un mal control glicémico. Debido a esta relación entre las dos patologías un trabajo interdisciplinario resulta fundamental más aun por la repercusión en la calidad de vida del paciente.

\section{Contribuciones de autoría:}

AC participó en el diseño el estudio y obtención de datos. AC, RG, NA, PM y AA realizaron el análisis e interpretación de resultados. Todos los autores redactaron, revisaron y aprobación de la versión final del manuscrito.

Fuente de financiamiento: Autofinanciado.

Conflictos de interés: No existe conflicto de intereses en este manuscrito.

\section{REFERENCIAS}

1. Organización Mundial de la salud. Informe mundial sobre la diabetes. 2016, disponible en: http: //www.who.int/ diabetes/global-report/es/>

2. Shaw J. Diabetes, Sindrome metabólico y epidémica cardiovascular. Diabetes Voices. 2006; 51: 27-29

3. Centers for Disease Control and Prevention-2011. National diabetes fact sheet: National estimates and general information on diabetes and pre-diabetes in the United States, 2010. Atlanta, GA: US Department of Health and Human Services, Canters for Disease Control and Prevention.

4. American Diabetes Association. Standars of medical care in diabetes-2010. Diabetes Care 33(Suppl 1): 1161; erratum in diabetes care, 2010; 33:693.

5. Saremi A, Nelson RG, Tulloch-Reid M, Hanson RL, Sievers ML, Taylos GW, et al. Periodontal Disease and mortality in tipe 2 diabetes. Diabetes care, 2005; 28:2732

6. Shultis WA, Weil EJ, Looker HC, Curtis JM, Sholossman M, Genco RJ. Effect of periodontitis on overt nephoropathy and end stage renal disease in tipe 2 diabetes. Diabetes Care, 2007; 30:306-311.

7. Bissong M, Azodo CC, Agbor MA, Nkuo-Akenji T, Fon $P N$. Oral health status of diabetes mellitus patients in Southwest Cameroon.Odontostomatol Trop. 2015 Jun; 38(150):49-57.

8. Luo H, Pan W, Sloan F, Feinglos M, Wu B. Forty-Year Trends in Tooth Loss Among American Adults With and Without Diabetes Mellitus: An Age-Period-Cohort Analysis. Prev Chronic Dis. 2015 Dec 3;12:E211.

9. Kinanes DF, Attstrom R. Advances in the pathogenesis al periodontitis. Group B consensus repot of the Fifth European Workshop in Periodontology. J Clin Periodontol 2005; 32:130-131.

10. Eke PI, Dye BA, Wei L, Thornton-Evans GO, Genco RJ; James B, Gordon D, Roy P. Prevalence of periodontitis in adults in the United States: 2009 and 2010. J Dent Res. 2012 Oct; 91(10):914-20. Epub 2012 Aug 30.
11. Alagl AS. Periodontal abscess as a possible oral clinical sign in the diagnosis of undiagnosed diabetes mellitus of elderly in a dental clinic set up a 7-year cross-sectional study. J Investig Clin Dent. 2016 May 8. doi: 10.1111/jicd.12217.

12. Genco RJ. Current view of risk factors for periodontal disease. J Periodontol. 1996; 67:10411049

13. Preshaw PM: Diabetes and Periodontal Disease. Int Dent J 2008; 58:237-243

14. Soskolne WA, Klinger A. The relationship between periodontal diseases and diabetes: an overview. Ann Periodontol 2001; 6:91-98.

15. Lalla E, Kaplan S, Chang SM, Roth GA, Celenti Hinckley K, Greenberg E, Papapanou PN. Periodontal

16. infection profiles in type 1 diabetes. J Clin Periodontol. 2006 Dec; 33(12):855-62.

17. Gómez DM. Condiciones periodontales en pacientes con diagnostico de diabetes mellitus tipo 2 , atendidos en el programa de pacientes con enfermedades crónicas de la foscal (parte 2). Trabajo Doctoral. Universidad Santo Tómas, Bucaramanga, 2016

18. Ramos D. Presencia de bacilos negro pigmentantés en bolsas periodontales de pacientes diabéticos tipo 2 y su relación con el control glicémico. Rev. Estomatol. Herediana 2017 [Consultado 31/10/2017]; .27(1). Disponible en: http://www.scielo.org.pe/scielo.php? script=sci_arttext\&pid=S101943552017000100005\&ln $\mathrm{g}=\mathrm{es} \& \mathrm{nrm}=\mathrm{iso}$

19. Ziukaite, DE Slot, CM Cobb. Prevalence of diabetes among patients diagnosed with periodontitis: A retrospective cross-sectional study. International Journal of dental hygiene. 2017; 12280:1-7.

20. Amare Teshome, Asmare Yitayeh. The effect of periodontal therapy on glycemic control and fasting plasma glucose level in type 2 diabetic patients: Systematic review and meta-analysis. BCM Oral Health. 2017; 0249-1:1-11.

21. Min Li, Cheng Zhang Li. High glucose improves healing of periodontal wound by inhibiting proliferation and osteogenetic differentiation of human PDL cells. International Wound Journal.2016; 1742:1-5.

22. Khanuja PK, Narula SJ, Rajput R, Sharma K, Tewari

23. S. Association of periodontal disease with glycemic control in patients with type 2 diabetes in India population. Front. Med. 2017, 11(1): 110-119.

24. K kapellas, G Mejia, PM Bartod, MR Skilton. Periodontal Therapy and glycaemic control among individuals with type 2 diabetes: reflections from the PerioCardio study. Int J Dent Hygiene.2016; 10.1111:1-10.

25. Paul Eke, Liang Wei, Gina Evans, Luisa Borrell. Risk Indicators for Periodontitis in Us Adults: National Health and Nutrition Examination Survey. Journal of Periodontology.2016; 10.1902; 1-18.

26. Tarid Abdulkabbar, Faisal Al-sahaly, Mohammed Alkathami. Comparison of periodontal and peri-implant inflammatory parameters among patients with prediabetes, type 2 diabetes mellitus and nondiabetic controls. Acta Odontologica Scandinavica. 2017;19(01): 1-7. 